\title{
Anesthesia Alert-A Preoperative Safety Concept for the Surgeon
}

\author{
Bogdan Protyniak1, Paul Pearce1, Luciana Giambarberi' ${ }^{1}$, Atul Kumar ${ }^{2}$, \\ Michael A. Goldfarb' \\ ${ }^{1}$ Department of Surgery, Monmouth Medical Center, Long Branch, USA \\ ${ }^{2}$ School of Medicine, St. George's University, St. George's, Grenada \\ Email: Protyniak@gmail.com
}

Received 9 January 2015; accepted 10 April 2015; published 14 April 2015

Copyright (C) 2015 by authors and Scientific Research Publishing Inc.

This work is licensed under the Creative Commons Attribution International License (CC BY). http://creativecommons.org/licenses/by/4.0/

c) (i) Open Access

\begin{abstract}
Background: Advances in modern medicine and surgical technique have allowed patients with multiple comorbidities to undergo invasive surgery electively. This places additional stress on hospital resources to anticipate and deal with potential complications. We have introduced a preoperative safety concept called "Anesthesia Alert". The surgeon or preoperative interviewing anesthesiologist assigns an Anesthesia Alert when booking to denote possible difficulty with anesthesia induction or intubation. As a result, two anesthesiologists and fiber optic equipment are made available on the day of surgery. Methods: A retrospective study of patients from all surgical specialties who were assigned Anesthesia Alerts between January 2012 and November 2012. Records were analyzed for reasons requiring Anesthesia Alerts. Patient demographics, comorbidities, and perioperative complications were reviewed. Results: A total of 112 patients formed this study group. Difficult airway comprised the majority of Anesthesia Alerts (n = 75, 67.0\%). Hypertension was the most prevalent comorbidity among patients. There were only 7 patients $(6.3 \%)$ admitted to the ICU postoperatively and no mortalities. Conclusion: Perioperative patient safety is an evolving concept that requires a team approach amongst the surgeon, anesthesiologist, and operating room staff. An Anesthesia Alert raises awareness for possible complications during anesthesia induction as well as allocates resources to increase patient safety and avoid preoperative delays. Most importantly, this concept places the onus on the surgeon to alert the anesthesiologist for possible complications during induction and extubation.
\end{abstract}

\section{Keywords}

Difficult Airway, Anesthesia Complications, Operating Room Safety 


\section{Introduction}

Advances in modern medicine and surgical technique have obviated preoperative hospital admission for many invasive surgical procedures. Patients are often seen in the surgeon's office before an operation and may or may not undergo preoperative medical clearance. In some cases, the anesthesiologist encounters the patient immediately before elective surgery. If the patient undergoes preoperative evaluation by Anesthesia, it may be a different anesthesiologist who evaluates them the morning of surgery. This places anesthesia staff in a demanding situation during an unexpected difficult airway, increasing patient risk.

The American Society of Anesthesiologists' (ASA) recent practice advisory states that timing of the preanesthetic evaluation can be guided by both surgical invasiveness and severity of disease [1]. Patients undergoing procedures with low surgical invasiveness can be evaluated on the day of surgery, while patients with high severity of disease have to be evaluated before the day of surgery [1]. The practice advisory does not take into account that a patient may have a difficult airway unrelated to the severity of their disease. Furthermore, the practice advisory recognizes that limitations in resources available to a specific healthcare system may affect the timing of the preanesthetic evaluation [1]. Therefore, it is the responsibility of the surgeon to alert anesthesia prior to surgery so that appropriate resources can be allocated to ensure patient safety.

We sought to introduce the concept of "Anesthesia Alert" as it pertains to patient safety during induction of anesthesia. An Anesthesia Alert is initiated in the outpatient setting prior to surgery if the surgeon feels the patient has a difficult airway and/or will require additional anesthesia support. On the day of surgery, fiber-optic equipment and two anesthesiologists are present during induction and extubation to handle any potential complications. Our study describes a series of patients who had Anesthesia Alerts and their outcomes.

\section{Methods}

We retrospectively analyzed all patients who had Anesthesia Alerts at Monmouth Medical Center (MMC) from January 2012 to November of 2012. MMC is a 527-bed university-affiliated community hospital. This study was approved by the Institutional Review Board. Simple descriptive statistics were used.

The reason for an Anesthesia Alert was obtained from patient records. Demographic and hospital-related information acquired included age, sex, race, length of stay and ICU admission. This information was then further organized by surgical specialties including: general (foregut, hindgut, vascular, breast, soft tissue and other), urology, obstetrics and gynecology, neurosurgery and orthopedics. The following patient comorbidities were reviewed: obesity, hypertension, diabetes, coronary artery disease (CAD), stroke, chronic obstructive pulmonary disease (COPD), asthma, obstructive sleep apnea (OSA), and miscellaneous pulmonary diseases.

\section{Results}

\subsection{Demographics}

A total of 112 patients formed the study group. The mean age was 61.7 years (range 19-89) between 67 (59.8\%) women and 45 (40.2\%) men. Ninety-one patients were Caucasian (81.3\%), 13 (11.6\%) African American, and eight (7.1\%) other. Demographics were further organized by surgical specialty (Table 1 ).

The average length of stay of all specialties was 1.7 days (Table 1). The longest length of stay averaged three days for orthopedic surgery patients. There were no mortalities among the 112 patients studied. Seven patients (6.3\%) required ICU admission (Table 1). Those patients underwent the following procedures: exploratory laparotomy with lysis of adhesions, incarcerated hernia repair and reconstruction of abdominal wall, laparoscopic Roux-en-Y gastric bypass, carotid endarterectomy, burrhole for subdural hematoma, transsphenoidal resection of a pituitary tumor, and two endovascular abdominal aortic aneurysm repairs. All had a "difficult airway" Anesthesia Alert with an ASA score of 3. They were discharged home or to rehab in stable condition. Only two patients developed post-operative complications, one bradycardia and one atelectasis.

\subsection{Comorbidities}

Of the comorbidities, hypertension $(n=63,56.3 \%)$ was the most common. Others included: obesity $(n=28$, $25 \%)$, diabetes $(n=25,22.3 \%)$, CAD $(n=15,13.4 \%)$, asthma $(n=13,11.6 \%)$, OSA $(n=9,8 \%)$, COPD $(n=2$, $1.8 \%)$, stroke $(\mathrm{n}=2,1.8 \%)$ and miscellaneous pulmonary diseases $(\mathrm{n}=2,1.8 \%)$ (Figure 1$)$. 
Table 1. Summary of results from 112 patients with Anesthesia Alerts.

\begin{tabular}{ccccccc}
\hline Surgical Specialty & Total Patients & Age (Range) & Sex (M/F) & Race (W/B/O) & $\begin{array}{c}\text { Length of } \\
\text { Stay (Days) }\end{array}$ & $\begin{array}{c}\text { ICU } \\
\text { Admissions }\end{array}$ \\
\hline General & $44(39.3 \%)$ & $65.8(19-89)$ & $15 / 29$ & $40 / 32 / 6$ & 1.5 & 5 \\
Urology & $14(12.5 \%)$ & $64.8(51-78)$ & $9 / 5$ & $12 / 1 / 1$ & 1.1 & 0 \\
OBGYN & $16(14.3 \%)$ & $53.7(25-76)$ & $0 / 16$ & $14 / 2 / 0$ & 0.6 & 0 \\
Neurosurgery & $6(5.4 \%)$ & $61.0(51-70)$ & $4 / 2$ & $6 / 0 / 0$ & 2.7 & 3 \\
Orthopedics & $32(28.5 \%)$ & $63.3(47-87)$ & $17 / 15$ & $28 / 3 / 1$ & 3 & 0 \\
\hline
\end{tabular}

${ }^{\mathrm{a}} \mathrm{W}$ : White; B: Black; O: Other.

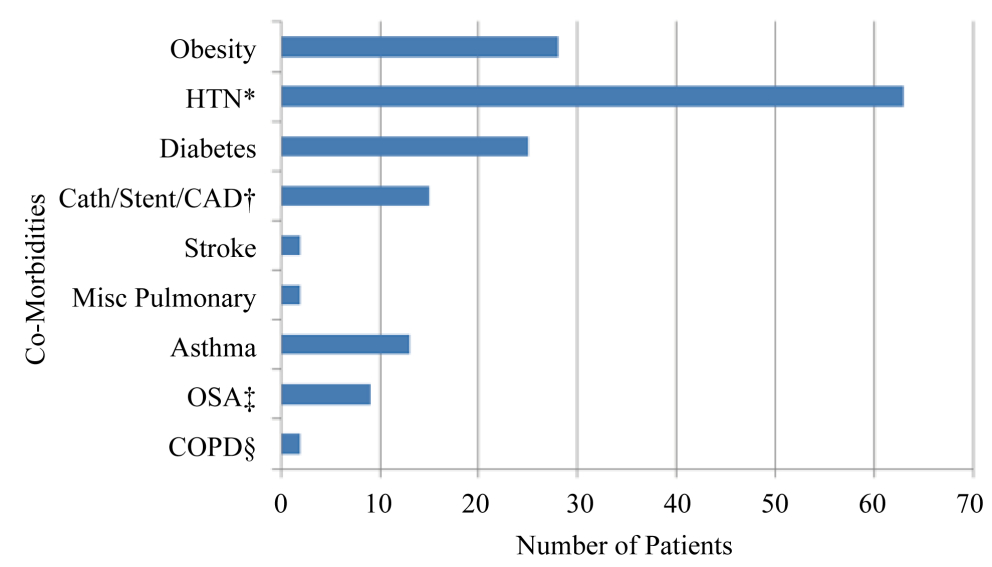

Figure 1. Comorbidities of the 112 patients evaluated for study. *HTN: hypertension; †Cath/Stent/CAD: coronary catheterization/coronary stent/coronary artery disease; $¥ O S A$ : obstructive sleep apnea; §COPD: chronic obstructive pulmonary disease.

\subsection{Anesthesia Alert}

The main reason for an Anesthesia Alert was “difficult airway” ( $n=103,92 \%)$. Nine patients (8\%) had Anesthesia Alerts categorized as “other”, including: myasthenia gravis, Guillain-Barre, fibromuscular dysplasia, muscular dystrophy, malignant hyperthermia, carcinoid syndrome, and allergy to anesthetic preservatives. The majority of American Society of Anesthesiologists (ASA) scores were 2 ( $\mathrm{n}=47,42 \%)$ and $3(\mathrm{n}=63,56.3 \%)$ (Figure 2).

\section{Discussion}

Ensuring the safety of all patients from arrival to departure the day of surgery is a team effort between nursing staff, anesthesiologists, and surgeons. While many protocols have been developed to ensure patient safety, there is a paucity of data that relates to evaluating the patient preoperatively in terms of any difficulty that may arise with induction of anesthesia. The ASA's Task Force on preanesthetic evaluation released a practice advisory aimed to assist in the decision making process in areas of patient care [1]. These practice advisories are not supported by scientific literature to the same degree as standards or guidelines because of the lack of sufficient evidence [1]. The Task Force believes that it is the obligation of the health care system to provide pertinent information to the anesthesiologist for the appropriate assessment of the severity of medical condition and invasiveness of procedure [1]. The Task Force, however, does not propose as to how this should be carried out. Furthermore, it acknowledges that certain healthcare systems have limitations in resources, which may affect the timing of the preanesthetic evaluation [1]. Our concept of Anesthesia Alert solves these two specific problems in that it places the onus on the surgeon to signal anesthesia well in advance so that appropriate resources and safety measures can be allocated in order to avoid perioperative delays and complications. 


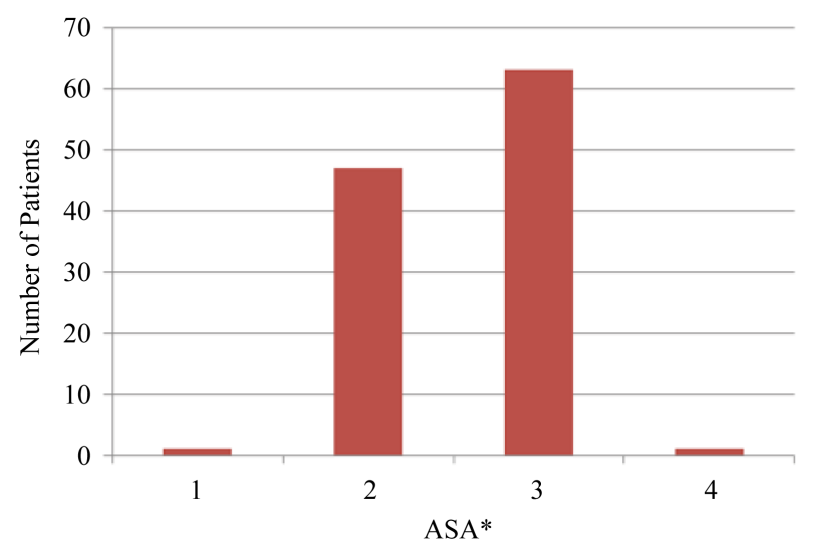

Figure 2. ASA classification of the 112 patients evaluated in the study. ${ }^{*}$ ASA: American Society of Anesthesiologists.

In our study, $92 \%$ of anesthesia alerts were due to a difficult airway. Problems with tracheal intubation are the main cause of hypoxemic anesthetic death and brain injury [2]. For the past three decades, the anesthesia literature has described poor airway management as a serious patient safety concern, requiring vigilant airway evaluation prior to induction of anesthesia [3]. The ASA task force on the management of the difficult airway describes it as a clinical situation in which a conventionally trained anesthesiologist experiences difficulty with facemask ventilation of the upper airway, difficulty with tracheal intubation, or both [4].

A recent meta-analysis of 35 studies (50,760 patients) found an overall incidence of difficult intubation to be $5.8 \%$ [5]. The incidence of unexpected difficult airway in clinical practice has not decreased despite advances in anesthesia protocols, devices, and patient monitoring [3]. Currently available screening tests for difficult intubation have only poor to moderate discriminative power when used alone [5]. These tests included: the Mallampati oropharyngeal classification, thyromental distance, sternomental distance, mouth opening, and Wilson risk score. Combinations of tests add some incremental diagnostic value in comparison to the value of each test alone, however, the clinical value of bedside screening tests for predicting difficult intubation remains limited [5]. A recent study by Cattano et al. in March 2013 showed that the use of a comprehensive airway assessment in 3460 preoperative patient encounters did not improve anesthesia resident ability to predict a difficult airway [3]. A complete airway assessment form using 11 of the ASA's proposed airway risk predictors improved documentation of airway assessment, but not the accuracy of such predictions [3].

Our concept of Anesthesia Alert requires the operating surgeon or the anesthesiologist screener to suspect and trigger the diagnosis of a difficult airway. Surgeons may not be as familiar as anesthesiologists with the list of difficult airway screening tests, however, surgeons have a pre-existing rapport with the patient. This rapport includes a thorough and often serial physical exam with follow-up. Any subtle and relevant clinical findings are more likely to be diagnosed by the surgeon and aid in triggering an Anesthesia Alert. Current literature shows that a difficult airway can easily be missed with established screening tests. Furthermore, based on the ASA's preanesthesia evaluation practice advisory, hospitals with limited resources may schedule an invasive procedure with anesthesia evaluation on the day of surgery. This gives the anesthesiologist insufficient time to prepare if a difficult airway is suspected, resulting in case delay and/or inability to properly handle unexpected complications.

The ASA's task force on management of the difficult airway published updated practice guidelines in February 2013 [4]. Their recommendations for difficult airway preparation include: 1) dedicated equipment readiness; 2) notifying the patient with an identified or alleged difficult airway; 3) allocating an individual to offer support; 4) preoxygenation via facemask; 5) administration of supplemental oxygen [4]. Despite these guidelines, the authors acknowledge that the literature is insufficient to evaluate the benefits of assigning an individual to provide assistance when a difficult airway is encountered. Furthermore, the guidelines do not specify whether the assistant should be a physician, nurse, or operating room technician. Our Anesthesia Alert concept mandates a second anesthesiologist to assist with intubation and extubation.

As modern medicine has advanced, it has allowed surgeons to operate on more patients with an increasing number of comorbidities. In our study the most common comorbidity that had the potential to affect the patient 
on the day of surgery was hypertension (Figure 1). Patients with uncontrolled hypertension run the risk of having their case cancelled on the day of surgery resulting in a delay of surgical treatment. Twenty five percent of the patients in our study also presented with morbid obesity. This factor in of itself will often make a patient a candidate for an Anesthesia Alert due to the number of issues that pertain to caring for a morbidly obese patient in the perioperative setting. The difficulties include intubating and ventilating the patients due to the excess of chest wall adipose tissue, a short neck, and airway obstruction. Many of our patients who received an Anesthesia Alert had comorbidities with an ASA score of 2 and 3, and underwent invasive elective procedures (Figure 2). Despite this, only seven patients (6.3\%) had to be admitted for monitoring in the ICU. All seven patients had an Anesthesia Alert for difficult airway. There were no mortalities in our study group.

While this study elucidates factors that should bring about an Anesthesia Alert there are limitations to this study. The short duration of this study limits the number of patients and various complications. There is no control group, thus comparisons to complications, delays, or time saved cannot be made based on this study. Anesthesia Alert has been readily adopted in all MMC departments where invasive procedures are performed.

\section{Conclusion}

Perioperative patient safety is an evolving concept that requires a team approach amongst the surgeon, anesthesiologist, and operating room staff. Given the recent constraints placed on healthcare, some hospitals may have limited resources. These become even harder to allocate in an emergency situation. The Anesthesia Alert safety concept calls on the surgeon to become an active participant in the preoperative anesthesia evaluation. Moreover, it pre-emptively allocates resources, such as an additional anesthesiologist, to maximize patient safety. Further studies will have to be performed to see what kind of an impact an Anesthesia Alert makes on patient outcomes.

\section{References}

[1] Committee on Standards and Practice Parameters, Apfelbaum, J.L., Connis, R.T., Nickinovich, D.G.; American Society of Anesthesiologists Task Force on Preanesthesia Evaluation, Pasternak, L.R., et al. (2012) Practice Advisory for Preanesthesia Evaluation: An Updated Report by the American Society of Anesthesiologists Task Force on Preanesthesia Evaluation. Anesthesiology, 116, 522-538. http://dx.doi.org/10.1097/ALN.0b013e31823c1067

[2] Henderson, J.J., Popat, M.T., Latto, I.P. and Pearce, A.C., Difficult Airway Society (2004) Difficult Airway Society Guidelines for Management of the Unanticipated Difficult Intubation. Anaesthesia, 59, 675-694. http://dx.doi.org/10.1111/j.1365-2044.2004.03831.x

[3] Cattano, D., Killoran, P.V., Iannucci, D., Maddukuri, V., Altamirano, A.V., Sridhar, S., et al. (2013) Anticipation of the Difficult Airway: Preoperative Airway Assessment, an Educational and Quality Improvement Tool. British Journal of Anaesthesia, 111, 276-285. http://dx.doi.org/10.1093/bja/aet029

[4] Apfelbaum, J.L., Hagberg, C.A., Caplan, R.A., Blitt, C.D., Connis, R.T., Nickinovich, D.G., et al. (2013) Practice Guidelines for Management of the Difficult Airway: An Updated Report by the American Society of Anesthesiologists Task Force on Management of the Difficult Airway. Anesthesiology, 118, 251-270. http://dx.doi.org/10.1097/ALN.0b013e31827773b2

[5] Shiga, T., Wajima, Z., Inoue, T. and Sakamoto, A. (2005) Predicting Difficult Intubation in Apparently Normal Patients: A Meta-Analysis of Bedside Screening Test Performance. Anesthesiology, 103, 429-437. 